\title{
Diagnosing lower gastrointestinal tuberculosis: colonoscopy is essential
}

\author{
Vui Heng Chong
}

Accepted: 17 November 2009/Published online: 11 December 2009

(C) Springer-Verlag 2009

\section{Dear Editor:}

I read with interest the publication on lower gastrointestinal tuberculosis (LGITB) by Lin et al. published in the October issue of the journal [1]. What they had shown is generally comparable to what have been reported. However, I am surprised that just over half $(n=15 / 28)$ of their patients had undergone colonoscopy evaluations. Considering the median age of their patients with most patients having symptoms, colonoscopy would have been indicated in most, if not all of their patients. As stated by the authors, if these patients had undergone colonoscopy, surgeries could have been avoided with the exception of few cases. For the two patients with perforations, urgent surgeries would have been unavoidable. However, for the remaining patients, including those with acute bleeding or bowel obstructions, most could have been initially managed conservatively. In fact, most would have settled down allowing for further evaluations to be carried out.

In our local setting, our surgical colleagues always prefer colonoscopic evaluations whenever possible even in those presenting with bowel obstructions or bleeding. Fortunately, catastrophic bleeding requiring immediate surgeries, including those secondary to LGITB are extremely rare. We are also well aware that colonic manifestations are varied and can be confused with inflammatory bowel disease and

\section{H. Chong $(\triangle)$}

Gastroenterology Unit, Department of Medicine,

Raja Isteri Pengiran Anak Saleha (RIPAS) Hospital,

Bandar Seri Begawan, BA 1710, Brunei Darussalam

e-mail: chongvuih@yahoo.co.uk colorectal cancer. However, this is not a major problem as biopsies are invariably obtained if any abnormalities are encountered. Most patients with LGITB that we encounter usually present at later stages of their diseases but we also need to remember that in some cases, the manifestations can be very mild. We have encountered cases where the abnormalities consisted of only non-specific inflammation and inconspicuous ileal polyp. Manifestations as diminutive colonic polyp have also been reported. Such patients, the diagnoses are usually unexpected and incidental. Overall, colonoscopy evaluation is an important aspect of evaluation of LGITB and should be considered even in patients with acute presentations. However, I do agree with Lin et al.'s remark that clinicians' suspicion is very important. It is usually the driving force that results in early diagnosis of LGITB. Clinicians' awareness of the different manifestations will certainly be helpful. Apart from all these, the major hurdle seems to be the difficulty with getting early colonoscopic examinations due to long waiting time in most centers. Despite busy schedules, we are usually able to arrange urgent colonoscopy evaluations within $24 \mathrm{~h}$. However, this requires good collaborations between the surgeons and the endoscopists and in most cases, these are achievable.

\section{References}

1. Lin PY, Wang JY, Hsueh PR, Lee LN, Hsiao CH, Yu CJ, Yang PC (2009) Lower gastrointestinal tract tuberculosis: an important but neglected disease. Int J Colorectal Dis 24:1175-1180 